\title{
Renewal Theorem for a Class of Stationary Sequences
}

\author{
S.P. Lalley \\ Department of Mathematical Statistics, Columbia University, New York, NY 10027, USA
}

Summary. A renewal theorem is obtained for stationary sequences of the form $\xi_{n}=\xi\left(\ldots, X_{n-1}, X_{n}, X_{n+1}, \ldots\right)$, where $X_{n}, n \in \mathbb{Z}$, are i.i.d. r.v.s. valued in a Polish space. This class of processes is sufficiently broad to encompass functionals of recurrent Markov chains, functionals of stationary Gaussian processes, and functionals of one-dimensional Gibbs states. The theorem is proved by a new coupling construction.

\section{Introduction}

Let $\xi_{1}, \xi_{2}, \ldots$ be a sequence of i.i.d. nonnegative random variables with finite expectation and a nonarithmetic distribution. A well-known consequence of Blackwell's renewal theorem is that if $S_{n}=\xi_{1}+\xi_{2}+\ldots+\xi_{n}$ and $\tau(b)$ $=\min \left\{n: S_{n}>b\right\}$, then $S_{\tau(b)}-b$ converges in distribution as $b \rightarrow \infty$.

The main result of this paper is a generalization of the aforementioned theorem to certain stationary sequences of random variables $\xi_{1}, \xi_{2}, \ldots$. The class of stationary sequences considered encompasses a large number of interesting special cases, including (a) $\xi_{n}=\xi\left(X_{n}\right)$, where $X_{n}$ is a stationary, recurrent Markov chain on a Polish space; (b) $\xi_{n}=\xi\left(T^{n} X\right)$, where $T$ is an ergodic automorphism of a compact abelian group and $X$ is uniformly distributed; (c) $\xi_{n}$ a stationary Gaussian sequence with a $C^{1}$ spectral density; and (d) $\xi_{n}=\xi\left(T^{n} X\right)$, where $T$ is the forward shift operator and $X$ is distributed on $\{1,2, \ldots, k\}^{\mathbb{Z}}$ according to a Gibbs measure.

Renewal theorems for various classes of stationary sequences have been proved by a number of authors. For functions of Markov chains, renewal theorems have been established by Orey (1961), Jacod (1971), Kesten (1974), and Athreya, Mac Donald, and Ney (1978). For flows over the shift (example (d) above) a theorem much stronger than the renewal theorem has been proved by Ratner (1978). Berbee (1979) gives a number of renewal theorems for

* Research supported by the National Science Foundation 
stationary sequences, but under stringent Markov-like conditions which rule out most of the examples mentioned above.

The approach taken in this paper is based on a coupling construction. The main thrust of the argument is similar to that of Lalley (1984); the primary novelty is in the construction of the coupling times.

\section{Statement of Principal Results}

Let $X_{n}, n \in \mathbb{Z}$, be a stationary, ergodic, $\chi$-valued sequence defined on a probability space $(\Omega, \mathscr{F}, P)$, where $\chi$ is a complete, separable, metric space. We shall use the boldface notation $\mathbf{X}_{m}$ to denote the entire sequence $X_{n}$ shifted $m$ units forward: thus the $n^{\text {th }}$ coordinate of $\mathbf{X}_{m}$ is $X_{m+n}$. We shall also use the boldface notation for other $\chi$-valued sequences of random variables or constants, e.g., $\mathbf{X}_{m}^{\prime}, \mathbf{x}_{m}$.

Suppose $\xi: \chi^{\mathbb{Z}} \rightarrow \mathbb{R}$ is a measurable function. Define real-valued random variables

$$
\begin{aligned}
\xi_{n} & =\xi\left(\mathbf{X}_{n}\right) & & (n \in \mathbb{Z}) \\
S_{n} & =\xi_{1}+\ldots+\xi_{n} & & (n>0) \\
S_{0} & =0 & & \\
S_{n} & =-\left(\xi_{0}+\xi_{-1}+\ldots+\xi_{-n+1}\right) & & (n<0) \\
\tau(b) & =\inf \left\{n \in \mathbb{Z}: S_{n}>b\right\} & & (b \in \mathbb{R}) .
\end{aligned}
$$

The main results of this paper will concern the quantities $S_{\tau(b)}-b$ and $\mathbf{X}_{\tau(b)}$ as $b \rightarrow \infty$. As in traditional renewal theory it is useful to allow for an "initial delay" in the system. Thus throughout the paper $S_{0}^{*}$ will be an arbitrary real random variable defined on $(\Omega, \mathscr{F})$, and

$$
\begin{aligned}
S_{n}^{*} & =S_{0}^{*}+S_{n} \quad(n \in \mathbb{Z}) \\
\tau^{*}(b) & =\inf \left\{n \in \mathbb{Z}: S_{n}^{*}>b\right\} \\
& =\tau\left(b-S_{0}^{*}\right) \quad(b \in \mathbb{R}) .
\end{aligned}
$$

Let

(1.3) $\mathscr{L}=\left\{\lambda: \chi^{\mathbb{Z}} \rightarrow[0, \infty)\right.$ Borel measurable, such that $\left.E \lambda\left(\mathbf{X}_{0}\right)=1\right\}$.

For each $\lambda \in \mathscr{L}$, define a probability measure $P_{\lambda}$ on $(\Omega, \mathscr{F})$

$$
\frac{d P_{\lambda}}{d P}=\lambda\left(\mathbf{X}_{0}\right)
$$

The main results of the paper will be valid for all of the probability measures $P_{\lambda}, \lambda \in \mathscr{L}$.

Special Flow under $\boldsymbol{\xi}>\mathbf{0}$ : If the function $\xi: \chi^{\mathbb{Z}} \rightarrow \mathbb{R}$ in (1.10) satisfies $\xi>0$ and $E \xi\left(\mathbf{X}_{0}\right)<\infty$, then the special flow under $\xi$ is defined to be the process

$$
\left(\mathbf{X}_{\tau^{*}(b)} ; S_{\tau^{*}(b)}^{*}-b\right), \quad b \geqq 0
$$


on the probability space $\left(\Omega, \mathscr{F}, P_{\lambda_{*}}\right)$, where

$$
\begin{aligned}
\lambda_{*}\left(\mathbf{X}_{0}\right) & =\xi\left(\mathbf{X}_{0}\right) / E \xi\left(\mathbf{X}_{0}\right), \\
S_{0}^{*} & =\xi_{0} U_{0},
\end{aligned}
$$

$U_{0}$ being independent of $X_{n}, n \in \mathbb{Z}$, and uniformly distributed on $(0,1)$. It is well-known (and easy to prove, cf. Cornfeld et al. (1982), Ch. 11, Lemma 1) that the special flow is stationary (in b) on the state space $\mathscr{R}(\xi)=\left\{(\mathbf{x}, s): \mathbf{x} \in \chi^{\mathbb{Z}}\right.$ and $0<s \leqq \xi(\mathbf{x})\}$.

Homology. Two functions $f, g: \chi^{\mathbb{Z}} \rightarrow \mathbb{R}$ (both measurable) are said to be "homologous" if there exists a measurable $\zeta: \chi^{\mathbb{Z}} \rightarrow \mathbb{R}$ such that

$$
f\left(\mathbf{X}_{0}\right)-g\left(\mathbf{X}_{0}\right)=\zeta\left(\mathbf{X}_{0}\right)-\zeta\left(\mathbf{X}_{1}\right) \quad \text { A.S. }(P)
$$

they are said to be " $L$-homologous" if there exists $\zeta \in L^{p}$ satisfying (1.7). If $f$ is $L^{p}$-homologous to zero then $f$ will be called $L^{p}$-null homologous; if $f$ is homologous to an integer-valued $g$, then $f$ will be called $\mathbb{Z}$-homologous.

Notice that homology is an equivalence relation. Also, if $f$ and $g$ are homologous by $\zeta$, then

$$
f\left(\mathbf{X}_{n}\right)-g\left(\mathbf{X}_{n}\right)=\zeta\left(\mathbf{X}_{n}\right)-\zeta\left(\mathbf{X}_{n+1}\right) \quad \text { A.S. }(P)
$$

for all $n \in \mathbb{Z}$, since $\left\{\mathbf{X}_{n}\right\}_{n \in \mathbb{Z}}$ is stationary under $P$.

Functions with Fading Memories. The sequence $\xi_{n}, n \in \mathbb{Z}$, will be said to have fading memory if

$$
\lim _{m \rightarrow \infty} E \sup _{n \geqq 1}\left|\left(S_{n+m}-S_{m}\right)-E\left(S_{n+m}-S_{m} \mid \mathscr{F}_{1}^{\infty}\right)\right|=0
$$

and

$$
\lim _{m \rightarrow \infty} E \sup _{n \geqq 1}\left|\left(S_{-n-m}-S_{-m}\right)-E\left(S_{-n-m}-S_{-m} \mid \mathscr{F}_{-\infty}^{0}\right)\right|=0
$$

where $S_{n}$ is as in (1.1) and

$$
\begin{aligned}
\mathscr{F}_{m}^{\infty} & =\sigma\left(X_{m}, X_{m+1}, X_{m+2}, \ldots\right), \\
\mathscr{F}_{-\infty}^{m} & =\sigma\left(X_{m}, X_{m-1}, X_{m-2}, \ldots\right) .
\end{aligned}
$$

Limiting Excess Distribution. If $E\left|\xi_{0}\right|<\infty$, define the limiting excess distribution $H$ by

$$
H(r ; F)=G(r ; F) / G\left(0 ; \chi^{\mathbb{Z}}\right)
$$

where

$$
G(r ; F)=\int_{r}^{\infty} P\left\{\max _{n<0} S_{n} \leqq-s ; \mathbf{X}_{0} \in F\right\} d s
$$


for real $r \geqq 0$ and Borel sets $F \subset \chi^{\mathbb{Z}}$. Notice that for each $F, H(r ; F)$ is continuous and nonincreasing in $r$. When $\xi>0$,

$$
H(r ; F)=\int_{r}^{\infty} P\left\{\xi_{0} \geqq s ; \mathbf{X}_{0} \in F\right\} d s / E \xi_{0}
$$

Theorem 1. Suppose that under $P$ the random variables $\left\{X_{n}\right\}_{n \in \mathbb{Z}}$ are i.i.d. Suppose also that $E\left|\xi_{0}\right|<\infty, E \xi_{0}>0, \xi_{n}$ has fading memory, and that no nonzero scalar multiple of $\xi$ is $\mathbb{Z}$-homologous. Then for every $r \in[0, \infty)$, all Borel cylinder sets $F_{0}, F_{1} \subset \chi^{\mathbb{Z}}$, every $\lambda \in \mathscr{L}$ and every initial delay variable $S_{0}^{*}$,

$$
\lim _{b \rightarrow \infty} P_{\lambda}\left\{S_{\tau^{*}(b)}^{*}-b>r ; \mathbf{X}_{\tau^{*}(b)} \in F_{1} ; \mathbf{X}_{0} \in F_{0}\right\}=H\left(r ; F_{1}\right) P_{\lambda}\left\{\mathbf{X}_{0} \in F_{0}\right\}
$$

The implicit hypothesis that the random variables $X_{n}$ take their values in a complete, separable metric space is probably extraneous. The only part of the proof where this is used is in Proposition 6 below; I suspect this may hold under the weaker assumption that $(\chi, \mathscr{P})$ is a separable measurable space.

Although Theorem 1 applies directly only to functionals $\xi(\cdot)$ of i.i.d. sequences $X_{n}$, it may be used indirectly to obtain renewal theorems for functionals of other ergodic sequences which contain "regeneration points". For example, a stationary, recurrent Markov chain on a denumerable state space may always be constructed by piecing together i.i.d. "words" of symbols from the state space. Athreya and Ney (1978) have shown that this may also be accomplished for certain Harris-recurrent chains on nondenumerable state spaces. Thus some of the standard renewal theorems for functionals of such Markov chains (cf. Athreya, Mac Donald, and Ney (1978)) may be recovered from our Theorem 1. Moreover, Theorem 1 applies to a much larger class of functionals: the theorems of Athreya et al. and their predecessors stipulate that the functional $\xi(\cdot)$ depend on only finitely many coordinates of the sequence $\mathbf{X}_{n}$.

Recently I have shown that regeneration points exist for a class of stationary processes known as "chains with complete connections" (cf. Lalley (1986)). A chain with complete connections is a stationary sequence $X_{n}, n \in \mathbb{Z}$, valued in a finite set $\chi$, whose distribution on $\chi^{\mathbb{Z}}$ is a Gibbs state in the sense of Bowen (1975). Many important dynamical systems, including ergodic automorphisms of compact groups, certain Anosov and Axiom $A$ diffeomorphisms of compact manifolds, certain measure-preserving maps of the unit interval, etc., may be "simulated" by chains with complete connections (cf. Bowen (1975) and Katznelson (1971)). Proposition 5 below shows that any function $\xi: \chi^{\mathbb{Z}} \rightarrow \mathbb{R}$ which is Hölder continuous (cf. the discussion following Proposition 5 below) gives rise to a sequence $\xi_{n}=\xi\left(X_{n}\right)$ with fading memory, provided $X_{n}$ is a chain with complete connections. Thus Theorem 1 applies to a large class of functionals on chains with complete connections, and, by extension, to functionals on those dynamical systems which may be simulated by a chain with complete connections.

Under what circumstances will a function $\xi: \chi^{\mathbb{Z}} \rightarrow \mathbb{R}$ not be $\mathbb{Z}$-homologous? Suppose $U_{0}$ is a random variable on $(\Omega, \mathscr{F}, P)$ which is uniformly distributed 
on $(0,1)$ and is independent of the stationary sequence $\left\{X_{n}\right\}_{n \in \mathbb{Z}}$. Let

$$
U_{n+1}=\left(U_{n}+\xi_{n+1}\right) \bmod 1 \quad \forall n \in \mathbb{Z} .
$$

Then the sequence $\left\{\left(X_{n}, U_{n}\right)\right\}_{n \in \mathbb{Z}}$ is stationary, as is easily verified: it will be referred to as the skew product sequence for the function $\xi$.

Proposition 1. If $\left\{X_{n}\right\}_{n \in \mathbb{Z}}$ is ergodic under $P$, then the skew product sequence is ergodic iff $\xi$ is not $\mathbb{Z}$-homologous.

This result is due to Anzai (1951) (cf. also Petersen (1983), Ch. 2, Theorem 4.8). For moving average processes, a sufficient condition is given in

Proposition 2. Suppose $\left\{a_{n}\right\}_{n \in \mathbb{Z}}$ is a sequence of real numbers such that

$$
\sum_{n}\left|a_{n}\right|<\infty \quad \text { and } \quad \sum_{n} a_{n} \neq 0 .
$$

Suppose $\left\{X_{n}\right\}_{n \in \mathbb{Z}}$ are i.i.d. $\mathbb{R}$-valued random variables with a nonlattice distribution (i.e., there do not exist $\alpha \neq 0, \beta$ such that $P\left\{\alpha X_{n}+\beta \in \mathbb{Z}\right\}=1$ ). Let

$$
\zeta\left(\mathbf{x}_{0}\right)=\sum_{n \in \mathbb{Z}} a_{n} x_{n} .
$$

Then no nonzero scalar multiple of $\xi$ is $\mathbb{Z}$-homologous.

Proposition 2 may be derived from Proposition 1: in fact it may be shown by a coupling argument that under the hypotheses of Proposition 2 the skew product sequence is mixing. Since this coupling argument is similar to the argument of Sect. 3 it is omitted.

Sufficient conditions for a sequence $\xi_{n}=\xi\left(\mathbf{X}_{n}\right)$ to have fading memory are given in the next three propositions.

Proposition 3. Let $X_{n}, n \in \mathbb{Z}$, be i.i.d. real-valued random variables with $E\left|X_{0}\right|<\infty$, and let

$$
\xi\left(\mathbf{x}_{0}\right)=\sum_{n=-\infty}^{\infty} a_{n} x_{n}
$$

for constants $a_{n}$ satisfying $\sum_{n=-\infty}^{\infty}\left|n a_{n}\right|<\infty$. Then

$$
\sum_{n=1}^{\infty} E\left|\xi_{n}-E\left(\xi_{n} \mid \mathscr{F}_{1}^{\infty}\right)\right|+\sum_{n=1}^{\infty} E\left|\xi_{-n}-E\left(\xi_{-n} \mid \mathscr{F}_{-\infty}^{0}\right)\right|<\infty .
$$

Proof. An easy calculation shows that

hence

$$
\xi_{n}-E\left(\xi_{n} \mid \mathscr{F}_{1}^{\infty}\right)=\sum_{k \leqq 0}\left(X_{k}-E X_{k}\right) a_{k-n}
$$

$$
\begin{aligned}
\sum_{n=1}^{\infty} E\left|\xi_{n}-E\left(\xi_{n} \mid \mathscr{F}_{1}^{\infty}\right)\right| & \leqq \sum_{k \leqq 0} E\left|X_{k}-E X_{k}\right| \sum_{n=1}^{\infty}\left|a_{k-n}\right| \\
& \leqq 2 E\left|X_{0}\right| \sum_{n=1}^{\infty} n\left|a_{-n}\right|<\infty
\end{aligned}
$$

A similar calculation gives the other inequality. 
Proposition 4. Let $f: \mathbb{R} \rightarrow \mathbb{R}$ be any uniformly Lipschitz continuous function. Suppose $\xi_{n}=\xi\left(X_{n}\right)$ satisfies

$$
\sum_{n=1}^{\infty} E\left|\xi_{n}-E\left(\xi_{n} \mid \mathscr{F}_{1}^{\infty}\right)\right|+\sum_{n=1}^{\infty} E\left|\xi_{-n}-E\left(\xi_{-n} \mid \mathscr{F}_{-\infty}^{0}\right)\right|<\infty .
$$

Then $\xi_{n}^{*}=f\left(\xi_{n}\right)$ has fading memory.

Proof. Since $f$ is uniformly Lipschitz continuous, there is a constant $C<\infty$ such that $|f(x)-f(y)| \leqq C|x-y|$ for all $x, y \in \mathbb{R}$. Hence

$$
\begin{aligned}
\mid f\left(\xi_{n}\right) & -E\left(f\left(\xi_{n}\right) \mid \mathscr{F}_{1}^{\infty}\right) \mid \\
& \leqq\left|f\left(\xi_{n}\right)-f\left(E\left(\xi_{n} \mid \mathscr{F}_{1}^{\infty}\right)\right)\right|+\left|E\left(f\left(E\left(\xi_{n} \mid \mathscr{F}_{1}^{\infty}\right)\right)-f\left(\xi_{n}\right) \mid \mathscr{F}_{1}^{\infty}\right)\right| \\
& \left.\leqq C\left|\xi_{n}-E\left(\xi_{n} \mid \mathscr{F}_{1}^{\infty}\right)\right|+C E\left(\mid \xi_{n}-E\left(\xi_{n} \mid \mathscr{F}_{1}^{\infty}\right)\right)|| \mathscr{F}_{1}{ }^{\infty}\right),
\end{aligned}
$$

so (1.9) follows easily from (1.21). A similar argument gives (1.10).

Let $\xi: \chi^{\mathbb{Z}} \rightarrow \mathbb{R}$ be Borel measurable; define

$$
\alpha_{n}(\xi)=\sup \left\{\left|\xi\left(\mathbf{x}_{0}\right)-\xi\left(\mathbf{x}_{0}^{\prime}\right)\right|: \mathbf{x}_{0}, \mathbf{x}_{0}^{\prime} \in \chi^{\mathbb{Z}} \text { such that } x_{m}=x_{m}^{\prime} \text { for }|m| \leqq n\right\} .
$$

Proposition 5. Let $X_{n}, n \in \mathbb{Z}$, be any stationary, ergodic, $\chi$-valued sequence, and let $\xi_{n}=\xi\left(\mathbf{X}_{n}\right)$. If $\sum_{n=1}^{\infty} \alpha_{n}(\xi)<\infty$, then (1.21) is satisfied.

Proof. It is easily verified that

$$
\begin{aligned}
E\left|\xi_{n}-E\left(\xi_{n} \mid \mathscr{F}_{1}^{\infty}\right)\right| \leqq \alpha_{n}(\xi), & n \geqq 1, \\
E\left|\xi_{n}-E\left(\xi_{n} \mid \mathscr{F}_{-\infty}^{0}\right)\right| \leqq \alpha_{n}(\xi), & n \leqq 0 .
\end{aligned}
$$

In the study of one-dimensional Gibbs states (cf. Bowen (1975)), $\chi$ is finite and of particular importance are the "Holder continuous" functions $\xi: \chi^{\mathbb{Z}} \rightarrow \mathbb{R}$, i.e., those for which $\alpha_{n}(\xi) \rightarrow 0$ at an exponential rate. By Propositions 4 and 5, such functions give rise to sequence $\xi_{n}=\xi\left(X_{n}\right)$ with fading memory.

Notice that if $\xi$ is any function on $\chi^{\mathbb{Z}}$ which depends on only finitely many coordinates then $\xi_{n}=\xi\left(\mathbf{X}_{n}\right)$ has fading memory. Thus Theorem 1 applies, provided the homology assumption is satisfied.

It follows from Propositions 2-4 that the hypotheses of Theorem 1 are satisfied for a large class of linear (moving average) processes. In particular, if $a_{n}, n \in \mathbb{Z}$ satisfy

and

$$
\sum_{n \in \mathbb{Z}}\left|n a_{n}\right|<\infty
$$

$$
\sum_{n \in \mathbb{Z}} a_{n} \neq 0,
$$

and if $X_{n}, n \in \mathbb{Z}$, are i.i.d. real-valued r.v.s. with a nonlattice distribution, then (1.15) holds for

$$
\xi_{m}=\sum_{n \in \mathbb{Z}} a_{n} X_{n+m},
$$


provided $E \xi_{0}>0$. It is well known that every stationary Gaussian sequence $\xi_{n}$ with $C^{1}$ spectral density has this form.

The rest of the paper is devoted to the proof of Theorem 1. Null homology is characterized in Sect. 2; coupling times are constructed in Sect. 3; ergodic theorems for the first-passage process are obtained in Sect. 4; and the connection between the existence of coupling times and the ergodic theorems is made in Sect. 5 , completing the circle.

The hypotheses of Theorem 1 will be in force throughout Sects. 3-5. In Sect. 2 we assume only that $X_{n}, n \in \mathbb{Z}$ is a stationary sequence with values in $\chi$, a complete separable metric space.

\section{Null Homology}

The notion of homology figures into the proof of Theorem 2 in two different ways. The fact that $\xi$ is not homologous to a constant is crucial in proving the existence of a coupling (cf. Sect. 3, Lemma 1). The stronger assumption that $\xi$ is not $\mathbb{Z}$-homologous enables us to use the ergodic theorem (cf. Proposition 1 and Proposition 8).

Proposition 6. Suppose $\xi: \chi^{\mathbb{Z}} \rightarrow \mathbb{R}$ is Borel measurable, satisfying

$$
E\left|\xi\left(\mathbf{X}_{0}\right)\right|=E\left|\xi_{0}\right|<\infty
$$

Then $\xi$ is $L^{1}$-null homologous iff the random variables $S_{n}=\sum_{j=1}^{n} \xi_{j}, n>0$, are $L^{1}$ -
bounded.

Proof. It is obvious that if $\xi$ is $L^{1}$-null homologous then the partial sums $\left\{S_{n}\right\}_{n \geqq 0}$ are $L^{1}$-bounded, because if $\xi_{n}=\zeta\left(\mathbf{X}_{n}\right)-\zeta\left(\mathbf{X}_{n+1}\right)$ then

$$
E\left|S_{n}\right|=E\left|\zeta\left(\mathbf{X}_{0}\right)-\zeta\left(\mathbf{X}_{n+1}\right)\right| \leqq 2 E\left|\zeta\left(\mathbf{X}_{0}\right)\right|
$$

Suppose now that $\left\|S_{n}\right\|_{1} \leqq 1$ for all $n \geqq 1$. For real $r, 0<r<1$, and $m \in \mathbb{Z}$, define

$$
W_{m}(r)=\sum_{n \geqq 0} \xi_{m+n} r^{n}
$$

Notice that this sum converges absolutely with probability one, since

$$
E\left(\sum_{n \geqq 0}\left|\xi_{n+m}\right| r^{n}\right)=\sum_{n \geqq 0} E\left|\xi_{n+m}\right| r^{n} \leqq \sum_{n \geqq 0} r^{n}
$$

Thus by Fubini's Theorem it is permissible to sum by parts, whence

$$
W_{1}(r)=\sum_{n=1}^{\infty} S_{n}\left(r^{n-1}-r^{n}\right)
$$

Since $\left\|S_{n}\right\|_{1} \leqq 1$ for all $n \geqq 1$, it follows immediately that $\left\|W_{1}(r)\right\|_{1} \leqq 1$ for all $0<r<1$. Moreover, since for each $r$ the process $\left\{W_{m}(r)\right\}_{m \in \mathbb{Z}}$ is stationary, it 
follows that

$$
\left\|W_{m}(r)\right\|_{1} \leqq 1 \quad \forall m \in \mathbb{Z}, \forall r, 0<r<1 .
$$

Now the set of $L^{1}$ random variables measurable with respect to $\sigma\left(\ldots, X_{-1}\right.$, $\left.X_{0}, X_{1}, X_{2}, \ldots\right)$ may be considered a subspace of $M\left(\chi^{\mathbb{Z}} \cup \Delta\right)$, the set of finite signed Borel measures on $\chi^{\mathbb{Z}} \cup \Delta$, the one-point compactification of $\chi^{\mathbb{Z}}$. Because of (2.2) and the separability of $\chi$, Helly's Selection Theorem implies that there exist $v \in M\left(\chi^{\mathbb{Z}} \cup \Delta\right)$ and a sequence $r_{k} \uparrow 1$ such that for every continuous function $f:\left(\chi^{\mathbb{Z}} \cup \Delta\right) \rightarrow \mathbb{R}$

$$
E f\left(\mathbf{X}_{0}\right) W_{0}\left(r_{k}\right) \rightarrow \int_{\chi^{\mathbb{Z}} \cup A} f d v
$$

Furthermore, according to the Lebesgue Decomposition and Radon-Nikodym theorems there exist a Borel-measurable $\zeta: \chi^{\mathbb{Z}} \rightarrow \mathbb{R}$ and a measure $v^{1} \in M\left(\chi^{\mathbb{Z}} \cup \Delta\right)$ such that

$$
\begin{aligned}
& E\left|\zeta\left(\mathbf{X}_{0}\right)\right|<\infty, \\
& v^{1} \text { is singular w.r.t. } \mathscr{D}\left(\mathbf{X}_{0}\right), \text { and } \\
& \int_{x^{\mathbb{Z} \cup \Delta}} f d v=E f\left(\mathbf{X}_{0}\right) \zeta\left(\mathbf{X}_{0}\right)+\int_{\chi^{\mathbb{Z}} \cup \Delta} f d v^{1}
\end{aligned}
$$

for every continuous function $f:\left(\chi^{\mathbb{Z}} \cup \Delta\right) \rightarrow \mathbb{R}$. We will conclude the proof by showing that

$$
\xi_{0}=\zeta\left(\mathbf{X}_{0}\right)=\zeta\left(\mathbf{X}_{0}\right)-\zeta\left(\mathbf{X}_{1}\right) \quad \text { A.S. }
$$

Observe that

$$
\begin{array}{r}
W_{0}(r)-W_{1}(r)=\xi_{0}+(r-1) W_{1}(r) \\
\stackrel{L^{1}}{\longrightarrow} \xi_{0} \quad \text { as } r \uparrow 1,
\end{array}
$$

in view of (2.2). Consequently, for any continuous $f:\left(\chi^{\mathbb{Z}} \cup \Delta\right) \rightarrow \mathbb{R}$,

$$
\begin{aligned}
E\left[f\left(\mathbf{X}_{0}\right)-f\left(\mathbf{X}_{-1}\right)\right] W_{0}(r) & =E f\left(\mathbf{X}_{0}\right)\left[W_{0}(r)-W_{1}(r)\right] \\
& \rightarrow E f\left(\mathbf{X}_{0}\right) \xi_{0}
\end{aligned}
$$

as $r \uparrow 1$. Now the map $\mathbf{x}_{0} \rightarrow f\left(\mathbf{x}_{0}\right)-f\left(\mathbf{x}_{-1}\right)(\Delta \rightarrow 0)$ is a continuous function on $\chi^{\mathbb{Z}} \cup \Delta$, since the backward shift map $\mathbf{x}_{0} \rightarrow \mathbf{x}_{-1}$ is a homeomorphism of $\chi^{\mathbb{Z}}$ which extends to a homeomorphism of $\chi^{\mathbb{Z}} \cup \Delta$ by $\Delta \rightarrow A$, so we may apply (2.3) to LHS (2.6) to obtain

$$
\begin{aligned}
& E\left[f\left(\mathbf{X}_{0}\right)-f\left(\mathbf{X}_{-1}\right)\right] W_{0}\left(r_{k}\right) \\
& \quad \rightarrow \int_{\chi^{\mathbb{Z} \cup \Delta}}\left[f\left(\mathbf{x}_{0}\right)-f\left(\mathbf{x}_{-1}\right)\right] v\left(d \mathbf{x}_{0}\right), \quad k \rightarrow \infty .
\end{aligned}
$$

Combining this with (2.4) and (2.6) yields 


$$
\begin{aligned}
E f\left(\mathbf{X}_{0}\right) \xi_{0}= & E\left[f\left(\mathbf{X}_{0}\right)-f\left(\mathbf{X}_{-1}\right)\right] \zeta\left(\mathbf{X}_{0}\right) \\
& +\int_{\chi^{\mathbb{Z} \cup \Delta}}\left[f\left(\mathbf{x}_{0}\right)-f\left(\mathbf{x}_{-1}\right)\right] v^{1}\left(d \mathbf{x}_{0}\right) \\
= & E f\left(\mathbf{X}_{0}\right)\left[\zeta\left(\mathbf{X}_{0}\right)-\zeta\left(\mathbf{X}_{1}\right)\right] \\
& +\int_{\chi^{\mathbb{Z} \cup \Delta}} f\left(\mathbf{x}_{0}\right)\left[v^{1}\left(d \mathbf{x}_{0}\right)-v^{1}\left(d \mathbf{x}_{1}\right)\right]
\end{aligned}
$$

for all continuous $f: \chi^{\mathbb{Z}} \cup \Delta \rightarrow \mathbb{R}$. But $v^{1}\left(d \mathbf{x}_{0}\right)$, and hence also $v^{1}\left(d \mathbf{x}_{0}\right)-v^{1}\left(d \mathbf{x}_{1}\right)$, are singular with respect to $\mathscr{D}\left(\mathbf{X}_{0}\right)$ : thus $(2.7)$ implies $v^{1}\left(d \mathbf{x}_{0}\right)=v^{1}\left(d \mathbf{x}_{1}\right)$ and

$$
E f\left(\mathbf{X}_{0}\right) \xi_{0}=E f\left(\mathbf{X}_{0}\right)\left[\zeta\left(\mathbf{X}_{0}\right)-\zeta\left(\mathbf{X}_{1}\right)\right]
$$

for all continuous $f: \chi^{\mathbb{Z}} \cup \Delta \rightarrow \mathbb{R}$. This proves (2.5), by the Riesz Representation Theorem.

There is a similar characterization of $L^{2}$-null homology:

Proposition 6'. Suppose $\xi: \chi^{\mathbb{Z}} \rightarrow \mathbb{R}$ is Borel measurable, satisfying $E\left|\xi_{0}\right|^{2}<\infty$. Then $\xi$ is $L^{2}$-null homologous iff the random variables $S_{n}=\sum_{j=1}^{n} \xi_{n}, n>0$, are $L^{2}$ -
bounded.

The proof is similar to that of Proposition 6, but easier, since one may use the $L^{2}$ weak topology and avoid the singular part $v^{1}$ altogether.

\section{Construction of Coupling Times}

The purpose of this section is to construct "coupling times", i.e., random times at which two random walks with different initial conditions meet and thereafter coalesce in some approximate sense. This construction differs from coupling constructions for Markov and semi-Markov processes (cf., for example, Lindvall (1977), Ney (1981), and Lalley (1984)) in that the Markov property cannot be used to arrange "coalescence" after meeting.

Recall that under the hypothesis of Theorem $1 X_{n}, n \in \mathbb{Z}$, are i.i.d. r.v.s. and that $\xi_{n}=\xi\left(\mathbf{X}_{n}\right)$ where

(3.1) $E\left|\xi_{0}\right|<\infty$;

(3.2) $\xi$ is not $L^{1}$-null homologous;

(3.3) $\xi_{n}$ has fading memory.

Proposition 7. There exists $h>0$ such that for every integer $k$, every integer $m_{0} \geqq 0$, and every real $\varepsilon>0$ there exist sequences $X_{n}^{\prime}, X_{n}^{\prime \prime}$ of $\chi$-valued r.v.s. and $a$ nonnegative, integer-valued random time $T=T(h k ; \varepsilon)$ satisfying

(3.4) the sequences $\left\{X_{n}^{\prime}\right\}_{n \in \mathbb{Z}}$ and $\left\{X_{n}^{\prime \prime}\right\}_{n \in \mathbb{Z}}$ are each identical in law to $\left\{X_{n}\right\}_{n \in \mathbb{Z}}$;

(3.5) $X_{m}^{\prime}=X_{m}^{\prime \prime} \quad \forall m \geqq T, \forall m \leqq m_{0}$;

$$
P\left\{\left|\sum_{n=1}^{m}\left(\xi\left(\mathbf{X}_{n}^{\prime}\right)-\xi\left(\mathbf{X}_{n}^{\prime \prime}\right)\right)-h k\right| \geqq \varepsilon \text { for some } m \geqq T\right\}<\varepsilon \text {. }
$$


The random walks $\sum_{n=1}^{m} \xi\left(\mathbf{X}_{n}^{\prime}\right)$ and $\sum_{n=1}^{m} \xi\left(\mathbf{X}_{n}^{\prime \prime}\right)+h k$ are coupled at time $T$ in the sense that with high probability they stay within $\varepsilon$ of each other after time $T$. The rest of this section is devoted to the proof of Proposition 7.

Suppose $X_{n}^{(a)}, n \in \mathbb{Z}$, and $X_{n}^{(b)}, n \in \mathbb{Z}$, are independent copies of the sequence $X_{n}, n \in \mathbb{Z}$. For each $A \subset \mathbb{Z}$ define

$$
\begin{aligned}
X_{n}(\Lambda) & =X_{n}^{(b)} \quad \text { if } n \in \Lambda, \\
& =X_{n}^{(a)} \quad \text { if } n \notin \Lambda \\
\xi_{n}(\Lambda) & =\xi\left(\mathbf{X}_{n}(\Lambda)\right) .
\end{aligned}
$$

Observe that for every $\Lambda \subset \mathbb{Z}$ the sequence $X_{n}(\Lambda)$ has the same distribution as the sequence $X_{n}$.

For $m \geqq 0$, define

$$
\begin{aligned}
\beta_{m}^{*} & =E \sup _{n \geqq 1}\left|\left(S_{n+m}-S_{m}\right)-E\left(\left(S_{n+m}-S_{m}\right) \mid \mathscr{F}_{0}^{\infty}\right)\right|, \\
\beta_{m}^{* *} & =E \sup _{n \geqq 1}\left|\left(S_{-n-m}-S_{-m}\right)-E\left(\left(S_{-n-m}-S_{-m}\right) \mid \mathscr{F}_{-\infty}^{0}\right)\right|, \\
\beta_{m} & =\beta_{m}^{*}+\beta_{m}^{* *} .
\end{aligned}
$$

By hypothesis, $\xi_{n}$ has fading memory, so $\beta_{m} \rightarrow 0$ as $m \rightarrow \infty$. By the triangle inequality and the stationarity of $X_{n}$,

provided

$$
E \sup _{n_{1}<k \leqq n_{2}}\left|\sum_{n=n_{1}}^{k}\left(\xi_{n}(\Lambda)-\xi_{n}\left(\Lambda^{*}\right)\right)\right| \leqq 16 \beta_{m}
$$

$$
n_{1}-m, n_{1}-m+1, n_{1}-m+2, \ldots, n_{2}+m \notin \Lambda \Delta \Lambda^{*} .
$$

(Here $\Delta$ denotes the symmetric difference of the two sets. It is possible that the constant 16 might be improved.)

Let $A_{m}=\{-m,-m+1, \ldots, m-1, m\}$.

Lemma 1. There exists a nonnegative integer $m_{*}$ such that

$$
\zeta=L^{1}-\lim _{k \rightarrow \infty} \sum_{n=-k}^{k}\left(\xi_{n}(\phi)-\xi_{n}\left(A_{m_{*}}\right)\right)
$$

exists and is not a.s. zero.

Notice that $E \zeta=0$, because each of the sums in (3.11) has a distribution which is symmetric about zero.

Lemma 1 depends heavily on the hypothesis that $\xi$ is not homologous to a constant. This is where the characterization of $L^{1}$-null homology given in Proposition 6 will be used.

Proof. By (3.10),

$$
E\left|\sum_{k<n \leqq m}\left(\xi_{n}(\phi)-\xi_{n}\left(A_{m_{*}}\right)\right)\right| \leqq 32 \beta_{k-m_{*}-1},
$$

provided $k>m_{*}$. Since $\beta_{n} \rightarrow 0$ as $n \rightarrow \infty$ it follows that the sums in (3.11) converge in $L^{1}$ regardless of how $m_{*}$ is chosen. 
To prove that $\zeta$ is not a.s. zero it suffices to show that $E|\zeta|>0$. First, observe that there is an integer $m_{1}$ sufficiently large that

$$
E\left|\sum_{n=-m_{2}}^{m_{2}}\left(\xi_{n}\left(\Lambda_{m_{1}+m_{2}}\right)-\xi_{n}(\mathbb{Z})\right)\right| \leqq i
$$

for every nonnegative integer $m_{2}$ : this follows from (3.10) and the fact that $\beta_{n} \rightarrow 0$ as $n \rightarrow \infty$. Next, notice that by the triangle inequality and Fatou's Lemma, together with (3.10),

$$
E\left|\sum_{|\boldsymbol{n}|>m_{2}}\left(\xi_{n}\left(\Lambda_{m_{1}+m_{2}}\right)-\xi_{n}(\phi)\right)\right| \leqq 16 m_{1} E\left|\xi_{0}\right|+32 \beta_{0}
$$

(write $\sum_{|n|>m_{2}}=\sum_{n \leqq-m_{1}-m_{2}}+\sum_{m_{2}<|n|<m_{1}+m_{2}}+\sum_{n \geqq m_{1}+m_{2}}$ ). The important feature of this inequality is that the bound doesn't depend on $m_{2}$. Finally, observe that

$$
\lim _{m_{2} \rightarrow \infty} E\left|\sum_{n=-m_{2}}^{m_{2}}\left(\xi_{n}(\phi)-\xi_{n}(\mathbb{Z})\right)\right|=\infty
$$

by Proposition 6 . Hence, by choosing $m_{2}$ sufficiently large and setting $m_{*}=m_{1}$ $+m_{2}$ we force $E|\zeta|>0$.

Proof of Proposition 7. Let $\left\{\left(X_{n}^{(a)}(k), X_{n}^{(b)}(k)\right)\right\}_{n \in \mathbb{Z}}, k=1,2, \ldots$, be independent sequences satisfying

$$
\begin{aligned}
& X_{n}^{(a)}(k)=X_{n}^{(b)}(k) \quad \forall|n|>m_{*} ; \\
& X_{n}^{(a)}(k), n \in \mathbb{Z}, \quad \text { and } \quad X_{n}^{(b)}(k), \quad|n| \leqq m_{*}, \quad \text { are i.i.d.; } \\
& \mathscr{D}\left(X_{n}^{(a)}(k)\right)=\mathscr{D}\left(X_{n}^{(b)}(k)\right)=\mathscr{D}\left(X_{0}\right) \quad \forall n \in \mathbb{Z},
\end{aligned}
$$

where $m_{*}$ is the integer provided by Lemma 1 . Lemma 1 implies that the r.v.s. $\zeta_{k}, k=1,2, \ldots$, defined by

$$
\zeta_{k}=\sum_{n=-\infty}^{\infty}\left(\xi\left(\mathbf{X}_{n}^{(a)}(k)\right)-\xi\left(\mathbf{X}_{n}^{(b)}(k)\right)\right)
$$

are i.i.d., with mean zero, but with nondegenerate distribution.

The plan is to construct $X_{n}^{\prime}, X_{n}^{\prime \prime}$ by splicing together segments of the sequences $X_{n}^{(a)}(k), X_{n}^{(b)}(k)$. Let

$$
\mathscr{S}_{k}=\sigma\left(X_{n}^{(a)}(j), X_{n}^{(b)}(j), n \in \mathbb{Z}, 1 \leqq j \leqq k\right), \quad k=1,2, \ldots,
$$

and let $t$ be a stopping time relative to the filtration $\mathscr{S}_{k}$. Choose integers $0 \leqq m_{0}<m_{1}<m_{2}<\ldots$ (where $m_{0}$ is as in the statement of Proposition 7) such that

$$
\beta_{m_{k}} \leqq \delta^{k}
$$

for a small $\delta>0$ : this is possible because by hypothesis $\xi_{n}$ has fading memory, hence $\beta_{m} \rightarrow 0$. Define integers $n_{1}, n_{2}, \ldots$, and $i(1), i(2), \ldots$ by

$$
\begin{aligned}
n_{1} & =4 m_{1}+2 m_{*}+1, \\
n_{k+1} & =n_{k}+4 m_{k+1}+2 m_{*}+1, \\
i(k) & =n_{k}-2 m_{k}-m_{*} .
\end{aligned}
$$


(a)

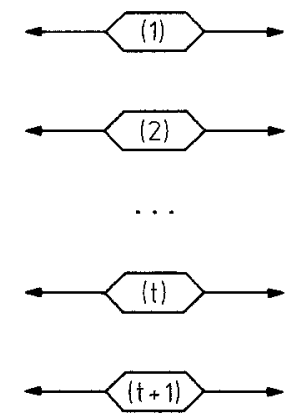

(b)

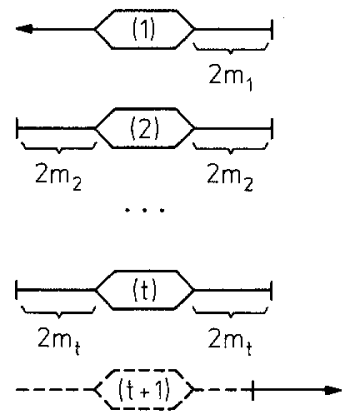

(c)

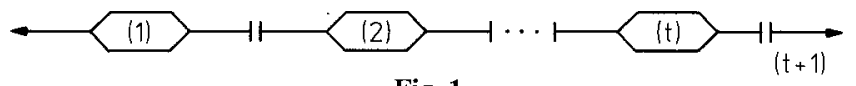

Fig. 1

Finally, define

$$
\begin{aligned}
& X_{n}^{\prime}=X_{n-i(1)}^{(a)}(1), \quad n \leqq n_{1}, \\
& =X_{n-i(k)}^{(a)}(k), \quad n_{k-1}<n \leqq n_{k}, \quad k \leqq t, \\
& =X_{n}^{(a)}(t+1), \quad n_{t}<n \text {; } \\
& X_{n}^{\prime \prime}=X_{n-i(1)}^{(b)}(1), \quad n \leqq n_{1} \text {, } \\
& =X_{n-i(k)}^{(b)}(k), \quad n_{k-1}<n \leqq n_{k}, \quad k \leqq t, \\
& =X_{n}^{(a)}(t+1), \quad n_{t}<n
\end{aligned}
$$

(cf. Fig. 1). Observe that $X_{n}^{\prime}=X_{n}^{\prime \prime}$ for $n \leqq m_{0}$ and $n>n_{t}$.

It is easily verified, using (3.12) and the fact that $t$ is a stopping time relative to the filtration $\mathscr{S}_{k}$, that the sequences $X_{n}^{\prime}$ and $X_{n}^{\prime \prime}$ are each identical in law to the sequence $X_{n}$. Moreover, since the sequences $X_{n}^{\prime}$ and $X_{n}^{\prime \prime}$ were created by independent substitutions, inequality (3.10) is applicable. Thus,

$$
\begin{aligned}
& E\left|\sum_{n=0}^{n_{1}-m_{1}}\left(\xi\left(\mathbf{X}_{n}^{\prime}\right)-\xi\left(\mathbf{X}_{n}^{\prime \prime}\right)\right)-\zeta_{1}\right| \leqq 32 \beta_{m_{1}} ; \\
& E\left|\sum_{n=n_{j}-m_{j}+1}^{n_{j+1}-m_{j+1}}\left(\xi\left(\mathbf{X}_{n}^{\prime}\right)-\xi\left(\mathbf{X}_{n}^{\prime \prime}\right)\right)-\zeta_{j+1}\right| \leqq 64 \beta_{m_{j+1}} \quad \text { if } t \geqq j+1 \quad \text { a.s.; } \\
& E \sup _{j \geqq 1}\left|\sum_{n=n_{k}-m_{k}+1}^{n_{k}-m_{k}+j}\left(\xi\left(\mathbf{X}_{n}^{\prime}\right)-\xi\left(\mathbf{X}_{n}^{\prime \prime}\right)\right)\right| \leqq 32 \beta_{m_{k}} \text { if } t=k \quad \text { a.s. }
\end{aligned}
$$

It follows that if $t$ is any stopping time satisfying $t \geqq 1$ a.s., then

$$
E\left|\sum_{n=n_{j}-m_{j}+1}^{n_{j+1}-m_{j+1}}\left(\xi\left(\mathbf{X}_{n}^{\prime}\right)-\xi\left(\mathbf{X}_{n}^{\prime \prime}\right)\right)-\zeta_{j+1}\right| 1\{t \geqq j+1\} \leqq 64 \beta_{m_{j+1}},
$$


and

$$
E \sup _{j \geqq 1}\left|\sum_{n=n_{\mathbf{t}}-m_{t}+1}^{n_{t}-m_{t}+j}\left(\xi\left(\mathbf{X}_{n}^{\prime}\right)-\xi\left(\mathbf{X}_{n}^{\prime \prime}\right)\right)\right| \leqq 32 \sum_{j=1}^{\infty} \beta_{m_{j}} .
$$

To finish the construction we must specify the stopping time $t$. Recall that by Lemma 1 the r.v.s. $\zeta_{1}, \zeta_{2}, \ldots$ are i.i.d., with mean zero, and with a nondegenerate distribution. Let $G$ be the smallest closed subgroup of $\mathbb{R}$ such that $P\left\{\zeta_{k} \in G\right\}=1$; for $g \in G$ and $\varepsilon_{*}>0$ define

$$
t=t\left(g, \varepsilon_{*}\right)=\min \left\{n \geqq 1:\left|\sum_{k=1}^{n} \zeta_{k}-g\right|<\varepsilon_{*}\right\} .
$$

By the well-known recurrence theorem for one-dimensional random walks, $t<\infty$ a.s. For a suitably small value of $\varepsilon_{*}>0$, define

$$
T=T(g ; \varepsilon)=n_{t\left(g, \varepsilon_{*}\right)}-m_{t\left(g, \varepsilon_{*}\right)}+1 \text {. }
$$

It is evident from the construction that (3.4) and (3.5) are valid. To verify (3.6), we observe that by (3.13)-(3.15)

$$
E \sup _{j \geqq 1}\left|\sum_{n=0}^{n_{t}-m_{t}+j}\left(\xi\left(\mathbf{X}_{n}^{\prime}\right)-\xi\left(\mathbf{X}_{n}^{\prime \prime}\right)\right)-\sum_{k=1}^{t} \zeta_{k}\right| \leqq 96 \sum_{j=1}^{\infty} \beta_{m_{j}} \leqq 96 \delta /(1-\delta) ;
$$

therefore, if $\delta>0$ and $\varepsilon_{*}>0$ are chosen sufficiently small, (3.6) must hold.

\section{Ergodic Theorem for First Passages}

Recall that $\tau(b), \tau^{*}(b)$ are the first passage times to $(b, \infty)$ by $S_{n}, S_{n}^{*}$ respectively (cf. (1.1) and (1.2)). Recall also that by Anzai's theorem (Proposition 1) the skew product sequence $\left(X_{n}, U_{n}\right), n \geqq 0$, is ergodic under the hypotheses of Theorem 1 . Finally, recall that $\mathscr{L}$ is the set of "likelihood ratios" with respect to $P$ (cf. (1.3)-(1.4)).

For real $r \geqq 0, b \in \mathbb{R}$, and Borel measurable $F \subset \chi^{\mathbb{Z}}$, define

$$
\begin{gathered}
J(b)=J(b ; r, F)=1\left\{S_{\tau(b)}-b>r ; \mathbf{X}_{\tau(b)} \in F\right\} \\
J^{*}(b)=J^{*}(b ; r, F)=1\left\{S_{\tau^{*}(b)}^{*}-b>r ; \mathbf{X}_{\tau^{*}(b)} \in F\right\} .
\end{gathered}
$$

The object of this section is to prove

Proposition 8. Suppose that under $P$ the skew product sequence $\left(X_{n}, U_{n}\right)$ is ergodic, that $E\left|\xi_{0}\right|<\infty, E \xi_{0}=\mu>0$, and that $S_{0}^{*} \geqq 0$ a.s. Then for each $\lambda \in \mathscr{L}$, $r \geqq 0, \delta>0$, and Borel measurable $F \subset \chi^{\mathbb{Z}}$,

$$
\lim _{m \rightarrow \infty} \sup _{b \geqq 0} P_{\lambda}\left\{\left|m^{-1} \sum_{k=1}^{m} J^{*}(b+k ; r, F)-H(r ; F)\right|>\delta\right\}=0 .
$$

The proof will proceed in two stages. First, it will be shown that the proposition is true under the additional assumption that $\xi_{n}>0$ a.s. Then it will be 
shown that by breaking the process up into "ladder epochs" one may deduce the general case from the case of positive increments.

\section{A. Positive Increments}

Assume that $\xi_{n}>0$ a.s. Recall that in this case $H(r ; F)$ is given by $(1.14)$. The ergodicity of the skew product will be used to establish

Lemma 2. Let $S_{0}^{*}=U_{0}$. Then for every $r=0, \delta>0$, and every Borel measurable $F \subset \chi^{\mathbb{Z}}$

$$
\lim _{m \rightarrow \infty} P\left\{\left|m^{-1} \sum_{k=1}^{m} J^{*}(k ; r, F)-H(r ; F)\right|>\delta\right\}=0 .
$$

Proof. Since $S_{n} / n \rightarrow \mu>0$ a.s., it follows that $\tau^{*}(m) / m \rightarrow \mu^{-1}$ a.s. Let $\varepsilon>0$; then on the event $\left\{(1-\varepsilon) \mu^{-1}<\tau^{*}(m) / m<(1+\varepsilon) \mu^{-1}\right\}$,

$$
\begin{aligned}
m^{-1} & \sum_{n: 1 \leqq n+1<m(1-\varepsilon) \mu^{-1}}\left[\left[\xi_{n+1}+U_{n}-r\right]\right]_{+} 1\left\{\mathbf{X}_{n+1} \in F\right\} \\
& \leqq m^{-1} \sum_{k=1}^{m} J^{*}(k ; r, F) \\
& \leqq m^{-1} \sum_{n: 1 \leqq n+1 \leqq m(1+\varepsilon) \mu^{-1}}\left[\left[\xi_{n+1}+U_{n}-r\right]\right]_{+} 1\left\{\mathbf{X}_{n+1} \in F\right\},
\end{aligned}
$$

where $[[\cdot]]$ denotes the greatest integer function. This is because in making a jump of size $\xi_{n+1}$ at time $n$ the random walk $S_{n}^{*}$ jumps over $\left[\left[\xi_{n+1}+U_{n}-r\right]\right]_{+}$ points $k$ by a distance of at least $r$.

Now, by the ergodicity of the skew product sequence and Birkhoff's ergodic theorem the extreme sides of the last inequality converge, as $m \rightarrow \infty$, to (1 $-\varepsilon) \mu^{-1} E\left[\left[\xi_{1}+U_{0}-r\right]\right]_{+} 1\left\{\mathbf{X}_{1} \in F\right\}$ and $(1+\varepsilon) \mu^{-1} E\left[\left[\xi_{1}+U_{0}-r\right]\right]_{+} 1\left\{\mathbf{X}_{1} \in F\right\}$, respectively. Since $U_{0}$ is independent of $\mathbf{X}_{0}$ and is uniformly distributed on $(0,1)$

$$
E\left[\left[\xi_{1}+U_{0}-r\right]\right]_{+} 1\left\{\mathbf{X}_{1} \in F\right\}=\mu H(r ; F),
$$

as an easy calculation shows. This proves (4.3).

Lemma 3. Let $S_{0}^{*}=U_{0}$. Then for every $r \geqq 0, \delta>0$, and $b \geqq 0$, and every Borel measurable $F \subset \chi^{\mathbb{Z}}$,

$$
\lim _{m \rightarrow \infty} P\left\{\left|m^{-1} \sum_{k=1}^{m} J^{*}(b+k ; r, F)-H(r ; F)\right|>\delta\right\}=0 .
$$

Proof. For $x \in \mathbb{R}$ let $\langle x\rangle$ denote the fractional part of $x$, i.e., $\langle x\rangle=x-[[x]]$. Let $U_{*}=\left\langle b-U_{0}\right\rangle$; since $U_{0}$ is independent of $\mathbf{X}_{0}$ and is uniformly distributed on $(0,1)$, so is $U_{*}$.

Now for any $b \geqq 0$,

$$
\left|m^{-1} \sum_{k=1}^{m} J^{*}(b+k ; r, F)-m^{-1} \sum_{k=1}^{m} J\left(k-U_{*} ; r, F\right)\right| \leqq m^{-1}[[2 b+2]],
$$


because the two sums differ only in the $[[2 b+2]]$ boundary terms. But Lemma 2 applies with $S_{0}^{*}=U_{*}$, giving

$$
\lim _{m \rightarrow \infty} P\left\{\left|m^{-1} \sum_{k=1}^{m} J\left(k-U_{*} ; r, F\right)-H(r ; F)\right|>\delta\right\}=0
$$

for all $\delta>0$. The result (4.4) follows.

Lemma 4. Let $\lambda \in \mathscr{L}$ and let $S_{0}^{*}$ be any nonnegative random variable. Then

iff

$$
\begin{array}{r}
\lim _{m \rightarrow \infty} P_{\lambda}\left\{\left|m^{-1} \sum_{k=1}^{m} J^{*}(b+k ; r, F)-H(r ; F)\right|>\delta\right\} \\
\forall r \geqq 0, \forall \delta>0, \forall b \geqq 0
\end{array}
$$

Similarly,

$$
\begin{array}{r}
\lim _{m \rightarrow \infty} P_{\lambda}\left\{\left|m^{-1} \sum_{k=1}^{m} J(b+k ; r, F)-H(r ; F)\right|>\delta\right\}=0 \\
\forall r \geqq 0, \forall \delta>0, \forall b \geqq 0 .
\end{array}
$$

iff

$$
\begin{gathered}
\lim _{m \rightarrow \infty} \sup _{b \geqq 0} P_{\lambda}\left\{\left|m^{-1} \sum_{k=1}^{m} J^{*}(b+k ; r, F)-H(r ; F)\right|>\delta\right\}=0 \\
\forall r \geqq 0, \forall \delta>0
\end{gathered}
$$

$$
\begin{gathered}
\lim _{m \rightarrow \infty} \sup _{b \geqq 0} P_{\lambda}\left\{\left|m^{-1} \sum_{k=1}^{m} J(b+k ; r, F)-H(r ; F)\right|>\delta\right\}=0 \\
\forall r \geqq 0, \forall \delta>0 .
\end{gathered}
$$

Consequently, if (4.5) (or (4.7)) holds for some nonnegative r.v. $S_{0}^{*}$, then it holds for every nonnegative r.v. $S_{0}^{*}$. Observe that by Lemma 3 (4.5) holds for every $S_{0}^{*} \geqq 0$ when $\lambda \equiv 1$. But clearly if (4.5) holds for $\lambda \equiv 1$ then it holds for every $\lambda \in \mathscr{L}$. Thus we have

Corollary 1. Let $\lambda \in \mathscr{L}$ and let $S_{0}^{*}$ be any nonnegative random variable. Then (4.5) holds.

Proof of Lemma 4. This is an easy consequence of the continuity in $r$ of $H(r ; F)$. The idea is to partition the probability space into the events

$$
G(i ; n)=\left\{i / n \leqq S_{0}^{*}<(i+1) / n\right\}
$$

where $n$ is so large that $H(r+2 / n ; F)-H(r-2 / n ; F)$ is small compared to $\delta$. On $G(i ; n)$ the random variable $J(b ; r, F)$ is bounded above and below by $J^{*}(b+(i$ $+1) / n ; r-1 / n)$ and $J^{*}(b+i / n ; r+1 / n, F)$, and similarly $J^{*}(b ; r, F)$ may be bounded above and below. The equivalence of (4.5) and (4.6), and of (4.7) and (4.8), follows by routine arguments based on these bounds.

The proof of Proposition 8 in the special case $\xi_{n}>0$ is now easily completed. By Lemma 4, to prove (4.2) it suffices to consider the special case $S_{0}^{*}$ $=\xi_{0} U_{0}$. Moreover, to prove (4.2) for arbitrary $\lambda \in \mathscr{L}$ it suffices to consider the 
special case $\lambda_{*}=\xi_{0} \mu^{-1}$ since $P_{\lambda} \ll P_{\lambda_{*}}$ for all $\lambda \in \mathscr{L}$. But if $S_{0}^{*}=\xi_{0} U_{0}$ then under $P_{\lambda_{*}}$ the process $\left(S_{\tau^{*}(b)}^{*}-b ; \mathbf{X}_{\tau^{*}(b)}\right), b \geqq 0$, is stationary (it is the "special flow under $\xi "$ defined in Sect. 1); thus,

$$
\begin{array}{r}
\sup _{b \geqq 0} P_{\lambda_{*}}\left\{\left|m^{-1} \sum_{k=1}^{m} J^{*}(b+k ; r, F)-H(r ; F)\right|>\delta\right\} \\
=P_{\lambda_{*}}\left\{\left|m^{-1} \sum_{k=1}^{m} J^{*}(k ; r, F)-H(r ; F)\right|>\delta\right\} .
\end{array}
$$

Therefore, (4.2) for $\lambda=\lambda_{*}$ and $S_{0}^{*}=\xi_{0} U_{0}$ follows from Corollary 1 .

\section{B. The General Case}

We shall reduce the general case to the special case considered in $4 \mathrm{~A}$. Define ladder indices $v(k), k \in \mathbb{Z}$, ladder increments $\xi_{k}^{\prime}, k \in \mathbb{Z}$, and ladder states $\left(X_{k}^{\prime}, U_{k}^{\prime}\right)$, $k \in \mathbb{Z}$ by

$$
\begin{aligned}
v(0) & =\max \left\{n \leqq 0: S_{n}>S_{n-i} \forall i \geqq 1\right\} ; \\
v(k+1) & =\min \left\{n>v(k): S_{n}>S_{v(k)}\right\}, \quad k \geqq 0 ; \\
v(k-1) & =\max \left\{n<v(k): S_{n}>S_{n-i} \forall i \leqq 1\right\}, \quad k \leqq 0 ; \\
\xi_{k}^{\prime} & =S_{v(k)}-S_{v(k-1)} ; \\
U_{k}^{\prime} & =U_{v(k)} ; \\
X_{k}^{\prime} & =\left(X_{1+v(k-1)}, X_{2+v(k-1)}, \ldots, X_{v(k)}\right) .
\end{aligned}
$$

Observe that the ladder indices are well-defined, because by the ergodic theorem $S_{n} \rightarrow+\infty$ as $n \rightarrow \infty$ and $S_{n} \rightarrow-\infty$ as $n \rightarrow-\infty$. Observe also that $U_{0}^{\prime}$ is uniform $(0,1)$ and is independent of $\left\{X_{k}^{\prime}\right\}_{k \in \mathbb{Z}}$, because $U_{0}$ is uniform $(0,1)$ and is independent of $\left\{X_{n}\right\}_{n \in \mathbb{Z}}$.

Next define a new probability measure $P^{\prime}$ on $(\Omega, \mathscr{F})$ by

$$
d P^{\prime} / d P=(v(1)-v(0))^{-1} / E(v(1)-v(0))^{-1} ;
$$

since $1 \leqq v(1)-v(0)<\infty, E(v(1)-v(0))^{-1}<\infty$, hence $P^{\prime}$ is well-defined, and the measures $P$ and $P^{\prime}$ are mutually absolutely continuous. Moreover, since the likelihood ratio $d P^{\prime} / d P$ is a function of the sequence $\left\{X_{n}\right\}_{n \in \mathbb{Z}}$,

$$
P^{\prime}\left(U_{0} \in d u \mid X_{n}, n \in \mathbb{Z}\right)=d u, \quad 0 \leqq u \leqq 1 .
$$

Lemma 5. Under $P^{\prime}$, the sequence $X_{k}^{\prime}, k \in \mathbb{Z}$, is stationary, and the skew product $\left(X_{k}^{\prime}, U_{k}^{\prime}\right)$ is ergodic. Also, $E^{\prime} \xi_{0}^{\prime}<\infty$.

The proof is fairly routine and is therefore omitted.

It follows from Lemma 5 and the result of Sect. 4A that Proposition 8 is valid for the sequence $X_{k}^{\prime}, \xi_{k}^{\prime}$, since $\xi_{k}^{\prime}>0$. But the first passage times $\tau^{*}(b)$ for the sums $S_{n}^{*}=S_{0}^{*}+\xi_{1}+\xi_{2}+\ldots+\xi_{n}$ lie in the sequence of ladder indices $v(k)$ ! Thus the general case follows from the special case. 


\section{Proof of Theorem 1}

The bulk of the argument will consist of showing that for every $r \in[0, \infty)$ and all Borel cylinder sets $F_{0}, F_{1} \subset \chi^{\mathbb{Z}}$,

$$
\lim _{b \rightarrow \infty} P\left\{S_{\tau(b)}-b>r ; \mathbf{X}_{\tau(b)} \in F_{1} ; \mathbf{X}_{0} \in F_{0}\right\}=H\left(r ; F_{1}\right) P\left\{\mathbf{X}_{0} \in F_{0}\right\}
$$

This uses the existence of coupling times and the ergodic theorem in a fashion similar to that in Sect. 3 of Lalley (1984).

Before proving (5.1) we shall indicate how one may deduce (1.15) from it. If (5.1) holds, then by an easy approximation argument it follows that for every $\lambda \in \mathscr{L}$

$$
\lim _{b \rightarrow \infty} P_{\lambda}\left\{S_{\tau(b)}-b>r ; \mathbf{X}_{\tau(b)} \in F_{1} ; \mathbf{X}_{0} \in F_{0}\right\}=H\left(r ; F_{1}\right) P_{\lambda}\left\{\mathbf{X}_{0} \in F_{0}\right\}
$$

This proves (1.15) in the special case $S_{0}^{*} \equiv 0$. If $S_{0}^{*}$ is a function of only finitely many of the r.v.s. $X_{n}$, then one may break up the probability space into the events

$$
G(i ; n)=\left\{(i / n) \leqq S_{0}^{*}<(i+1) / n\right\}
$$

and use (5.2) together with the continuity in $r$ of $H\left(r ; F_{1}\right)$ to get (1.15) (notice that each of the events $G(i ; n)$ is a cylinder set). Finally, in the general case, one may approximate $S_{0}^{*}$ by random variables depending on only finitely many of the r.v.s. $X_{n}$, and exploit again the continuity in $r$ of $H\left(r ; F_{1}\right)$ to deduce $(1.15)$. The details of these arguments are quite routine.

Thus, it remains to show (5.1). Observe that if the function $\xi$ satisfies the hypotheses of Theorem 1 then so does $\delta \xi$, for any constant $\delta>0$. Thus it suffices to prove Theorem 1 for some scalar multiple $\delta \xi, \delta>0$. In particular, we may (and shall) assume that $\xi$ has been rescaled so that $h=1$ in Proposition 7.

Proposition 7 (the existence of coupling times) will now be used to establish

Lemma 6. For each real $r \geqq 0$, each integer $k$, each $\varepsilon>0$, and all Borel cylinder sets $F_{0}, F_{1} \subset \chi^{\mathbb{Z}}$, there exists $b_{0}<\infty$ sufficiently large that whenever $b \geqq b_{0}$

$$
\begin{aligned}
& P\left\{S_{\tau(b-k-\varepsilon)}-(b-k-\varepsilon)>r+2 \varepsilon ; \mathbf{X}_{\tau(b-k-\varepsilon)} \in F_{1} ; \mathbf{X}_{0} \in F_{0}\right\} \\
& \quad-P\left\{S_{\tau(b-k-\varepsilon)}-(b-k-\varepsilon) \leqq 2 \varepsilon\right\}-2 \varepsilon \\
& \leqq P\left\{S_{\tau(b)}-b>r ; \mathbf{X}_{\tau(b)} \in F_{1} ; \mathbf{X}_{0} \in F_{0}\right\} \\
& \leqq P\left\{S_{\tau(b-k-\varepsilon)}-(b-k-\varepsilon)>r-2 \varepsilon ; \mathbf{X}_{\tau(b-k-\varepsilon)} \in F_{1} ; \mathbf{X}_{0} \in F_{0}\right\} \\
&+P\left\{S_{\tau(b-k-\varepsilon)}-(b-k-\varepsilon) \leqq 2 \varepsilon\right\}+2 \varepsilon .
\end{aligned}
$$

Proof. Assume that the event $\left\{\mathbf{X}_{0} \in F_{0}\right\}$ depends only on the coordinates $X_{n}$, $n<m_{0}$. By Proposition 7 there exist $X_{n}^{\prime}, X_{n}^{\prime \prime}$, and $T=T(k ; \varepsilon)$ such that (3.4)-(3.6) hold; hence

where

$$
P\left(\Gamma_{1}\right)>1-\varepsilon
$$

$$
\Gamma_{1}=\left\{\left|\sum_{n=1}^{m}\left(\xi\left(\mathbf{X}_{n}^{\prime}\right)-\xi\left(\mathbf{X}_{n}^{\prime \prime}\right)\right)-k\right|<\varepsilon \forall m \geqq T\right\} .
$$


Notice also that because of (3.5)

$$
\left\{\mathbf{X}_{0}^{\prime} \in F_{0}\right\}=\left\{\mathbf{X}_{0}^{\prime \prime} \in F_{0}\right\}
$$

Define

and let

$$
\begin{aligned}
\tau^{\prime}(b) & =\inf \left\{m \geqq 0: \sum_{n=1}^{m} \xi\left(\mathbf{X}_{n}^{\prime}\right)>b\right\}, \\
\tau^{\prime \prime}(b) & =\inf \left\{m \geqq 0: \sum_{n=1}^{m} \xi\left(\mathbf{X}_{n}^{\prime \prime}\right)>b-k\right\},
\end{aligned}
$$

$$
\Gamma_{2}=\left\{T+m_{1}<\min \left(\tau^{\prime}(b), \tau^{\prime \prime}(b)\right) \forall b \geqq b_{0}-\varepsilon\right\} .
$$

Here $m_{1}$ should be chosen sufficiently large that $\left\{\mathbf{X}_{0} \in F_{1}\right\}$ depends only on $X_{n}$, $|n|<m_{1}$, and $b_{0}$ should be sufficiently large that

Hence by (3.5)

$$
P\left(\Gamma_{2}\right)>1-\varepsilon \text {. }
$$

$$
\left\{\mathbf{X}_{k}^{\prime} \in F_{1}\right\}=\left\{\mathbf{X}_{k}^{\prime \prime} \in F_{1}\right\} \quad \forall k \geqq T+m_{1}
$$

Now on $\Gamma_{1} \cap \Gamma_{2}, \tau^{\prime}(b)=\tau^{\prime \prime}(b-\varepsilon)$ except possibly when

$$
\sum_{n=1}^{\tau^{\prime \prime}(b-\varepsilon)}\left(\xi\left(\mathbf{X}_{n}^{\prime \prime}\right)\right)-b+k \leqq 2 \varepsilon
$$

Consequently, on $\Gamma_{1} \cap \Gamma_{2}$

and

$$
\begin{aligned}
& \left\{\sum_{n=1}^{\tau^{\prime \prime}(b-\varepsilon)} \xi\left(\mathbf{X}_{n}^{\prime \prime}\right)-(b-k-\varepsilon)>r+2 \varepsilon\right\} \Rightarrow \\
& \left\{\sum_{n=1}^{\mathfrak{t}^{\prime}(b)} \xi\left(\mathbf{X}_{n}^{\prime}\right)-b>r\right\} \Rightarrow \\
& \left\{\sum_{n=1}^{t^{\prime \prime}(b-\varepsilon)} \xi\left(\mathbf{X}_{n}^{\prime \prime}\right)-(b-k-\varepsilon)>r-2 \varepsilon\right\} \text { or } \\
& \left\{\sum_{n=1}^{\tau^{\prime \prime}(b-\varepsilon)} \xi\left(\mathbf{X}_{n}^{\prime \prime}\right)-(b-k-\varepsilon) \leqq 2 \varepsilon\right\} ; \\
& \left\{\mathbf{X}_{0}^{\prime} \in F_{0}\right\}=\left\{\mathbf{X}_{0}^{\prime \prime} \in F_{0}\right\} ;
\end{aligned}
$$

$$
\left\{\mathbf{X}_{\tau^{\prime}(b)}^{\prime} \in F_{1}\right\}=\left\{\mathbf{X}_{\tau^{\prime \prime}(b-\varepsilon)}^{\prime \prime} \in F_{1}\right\} \quad \text { on } \quad\left\{\tau^{\prime}(b)=\tau^{\prime \prime}(b-\varepsilon)\right\}
$$

The inequalities (5.3) now follow from (3.4) and the fact that $P\left(\Gamma_{1} \cap \Gamma_{2}\right)>1$ $-2 \varepsilon$.

The proof of (5.1) now proceeds as follows. Choose $b_{0}$ so large that the inequalities (5.3) hold for all $b \geqq b_{0}$ and $k=1,2, \ldots, m$. Average these inequalities to obtain 


$$
\begin{aligned}
& E\left(m^{-1} \sum_{k=1}^{m} J\left(b-k-\varepsilon ; r+2 \varepsilon ; F_{1}\right) 1\left\{\mathbf{X}_{0} \in F_{0}\right\}\right) \\
& \quad-E\left(m^{-1} \sum_{k=1}^{m}\left(1-J\left(b-k-\varepsilon ; 2 \varepsilon ; \chi^{\mathbb{Z}}\right)\right)\right)-2 \varepsilon \\
& \leqq P\left\{S_{\tau(b)}-b>r ; \mathbf{X}_{\tau(b)} \in F_{1} ; \mathbf{X}_{0} \in F_{0}\right\} \\
& \leqq E\left(m^{-1} \sum_{k=1}^{m} J\left(b-k-\varepsilon ; r-2 \varepsilon ; F_{1}\right) 1\left\{\mathbf{X}_{0} \in F\right\}\right) \\
&+E\left(m^{-1} \sum_{k=1}^{m}\left(1-J\left(b-k-\varepsilon ; 2 \varepsilon ; \chi^{\mathbb{Z}}\right)\right)\right)+2 \varepsilon
\end{aligned}
$$

where $J(\cdot ; \cdot ; \cdot)$ is defined by (4.1). Now it follows from Proposition 8 that if $m$ is large then the extreme of this inequality are approximately

and

$$
H\left(r+2 \varepsilon ; F_{1}\right) P\left\{\mathbf{X}_{0} \in F_{0}\right\}+\left(1-H\left(2 \varepsilon ; \chi^{\mathbb{Z}}\right)\right)-2 \varepsilon,
$$

$$
H\left(r-2 \varepsilon ; F_{1}\right) P\left\{\mathbf{X}_{0} \in F_{0}\right\}+\left(1-H\left(2 \varepsilon ; \chi^{\mathbb{Z}}\right)\right)+2 \varepsilon .
$$

Letting $\varepsilon \rightarrow 0$ and using the continuity in $r$ of $H(r ; F)$, we obtain (4.1).

\section{References}

Anzai, H.: Ergodic skew product transformations on the torus. Osaka Math. J. 3, 83-99 (1951)

Athreya, K., McDonald, D., Ney, P.: Limit theorems for semi-Markoy processes and renewal theory for Markov chains. Ann. Probability 6, 788-797 (1978)

Athreya, K., Ney, P.: A new approach to the limit theory of recurrent Markov chains. Trans. Amer. Math. Soc. 245, 493-501 (1978)

Berbee, H.: Random Walks with Stationary Increments and Renewal Theory. Math. Centrum Tract 112, Amsterdam (1979)

Bowen, R.: Equilibrium States and the Ergodic Theory of Anosov Diffeomorphisms. Lecture Notes in Math. 470. Berlin Heidelberg New York: Springer 1975

Cornfeld, I., Fomin, S., Sinai, Y.: Ergodic Theory. Berlin Heidelberg New York: Springer 1982

Jacod, J.: Théorème de renouvellement et classification pour les chaines semi-Markoviennes. Ann. Inst. H. Poincaré B 7, 83-129 (1971)

Katznelson, Y.: Ergodic automorphisms of $T^{n}$ are Bernoulli shifts. Israel J. Math. 10, 186-195 (1971)

Kesten, H: Renewal theory for functionals of a Markov chain with general state space. Ann. Probability 2, 355-386 (1974)

Lalley, S.: Conditional Markov renewal theory I. Countable state space. Ann. Probability 12, $1113-1148$ (1984)

Lalley, S.: Regenerative representation for one-dimensional Gibbs states. Ann. Probability 14 (1986)

Lindvall, T.: A probabilistic proof of Blackwell's theorem. Ann. Probability 5, 482-485 (1977)

Ney, P.: A refinement of the coupling method in renewal theory. Stoch. Proc. Appl. 11, 11-26 (1981)

Orey, S.: Change of time scale for Markov processes. Trans. Am. Math. Soc. 99, 384-390 (1961)

Petersen, K.: Ergodic Theory. Cambridge: Cambridge Univ. Press 1983

Ratner, M.: Bernoulli flows over maps of the interval. Israel J. Math. 31, 298-314 (1978) 